\title{
Addressing intimate partner violence during the COVID-19 pandemic and beyond: how radiologists can make a difference
}

\author{
Simon Matoori ${ }^{1,2,3}$ (1) Bharti Khurana ${ }^{4} \cdot$ Marta Chadwick Balcom $^{5} \cdot$ Johannes M. Froehlich $^{2,6} \cdot$ Sonja Janssen $^{7}$. \\ Rosemarie Forstner ${ }^{3}$ - Ann D. King ${ }^{8}$ • Dow-Mu Koh ${ }^{9}$ • Andreas Gutzeit ${ }^{2,3,10}$
}

Received: 4 August 2020 / Revised: 14 September 2020 / Accepted: 21 September 2020 / Published online: 6 October 2020

(C) European Society of Radiology 2020

\begin{abstract}
Faced with the COVID-19 pandemic, many countries both in Europe and across the world implemented strict stay-at-home orders. These measures helped to slow the spread of the coronavirus but also led to increased mental and physical health issues for the domestically confined population, including an increase in the occurrence of intimate partner violence (IPV) in many countries. IPV is defined as behavior that inflicts physical, psychological, or sexual harm within an intimate relationship. We believe that as radiologists, we can make a difference by being cognizant of this condition, raising an alert when appropriate and treating suspected victims with care and empathy. The aim of this Special Report is to raise awareness of IPV among radiologists and to suggest strategies by which to identify and support IPV victims.

Key Points

- The COVID-19 pandemic led to a marked increase in the number of intimate partner violence (IPV) cases, potentially leading to increased emergency department visits and radiological examinations.

- Most IPV-related fractures affect the face, fingers, and upper trunk, and may easily be misinterpreted as routine trauma.

- Radiologists should carefully review the medical history of suspicious cases, discuss the suspicion with the referring physician, and proactively engage in a private conversation with the patient, pointing to actionable resources for IPV victims.
\end{abstract}

Keywords Intimate partner violence $\cdot$ Domestic violence $\cdot$ Radiology $\cdot$ Wounds and injuries $\cdot$ Coronavirus

\section{Abbreviations}

CT Computed tomography

ED Emergency department

IPV Intimate partner violence

LGBT Lesbian, gay, bisexual, and transgender

Simon Matoori

smatoori@seas.harvard.edu

$\triangle$ Andreas Gutzeit

andreas.gutzeit@hirslanden.ch

1 John A. Paulson School of Engineering and Applied Sciences, Harvard University, Cambridge, MA, USA

2 Institute of Radiology and Nuclear Medicine and Cancer Center St. Anna Klinik Luzern, Hirslanden Klinik St. Anna, Lucerne, Switzerland

3 Department of Radiology, Paracelsus Medical University, Salzburg, Austria

4 Department of Radiology, Brigham and Women's Hospital, Boston, MA, USA

\section{COVID-19: a catalyst for intimate partner violence}

In response to the COVID-19 pandemic, most governments of Asian, European, Australian, and American countries enacted nation-wide lockdowns ordering the closure

5 Community Health Intervention and Prevention Programs, Brigham and Women's Hospital, Boston, MA, USA

6 Clinical Research Group, Klus Apotheke Zurich, Zurich, Switzerland

7 Clinic of Radiology and Nuclear Medicine, University Medical Center Mannheim, University of Heidelberg, Mannheim, Germany

8 Department of Imaging and Interventional Radiology, The Chinese University of Hong Kong, Prince of Wales Hospital, Hong Kong, SAR, China

9 Department of Radiology, Royal Marsden Hospital, Sutton, UK

10 Department of Chemistry and Applied Biosciences, Institute of Pharmaceutical Sciences, ETH Zurich, Zurich, Switzerland 
of all schools, universities, and non-essential businesses, in addition to restricting public gatherings and recommending that people stay at home. While these interventions slowed the spread of the coronavirus to some degree, they brought with them a multitude of associated mental and physical health issues for the confined population [1-3]. As the pandemic is still ongoing, it is too early to make a judgement on what can be learned from it, or to measure the full impact of social distancing and lockdown. However, it has become evident that the pandemic is not only a medical challenge, but also strains entire social and political systems across the world. Radiologists are integral providers of medical care and encounter a wide cross-section of patients, including patients with injuries related to intimate partner violence (IPV). The incidence and severity of this form of violence has dramatically increased during the pandemic, with double-digit increases in incidence in several countries around the globe [4-7]. The COVID-19 pandemic has negatively affected IPV victims in many ways, constraining them to unsafe home environments and limiting both their access to and the availability of support services such as hospital-based support systems, counselling, and women's shelters.

As imaging professionals, we should consider our societal role during the pandemic. The concept of becoming involved in the social needs of patients may be unusual to some radiologists. When asked whether they should proactively inquire about the mechanism of trauma when they suspect IPV, many radiologists reply that the emergency department (ED) or referring physician should be responsible. But what happens if this is overlooked in a busy ED, especially during the pandemic? Will such injuries be treated as routine trauma? The victim may not know that resources are available to support IPV victims. The aims of this special report are to raise awareness among radiologists and allied health professionals as to what injuries are associated with IPV and how to proceed when IPV is suspected.

\section{Intimate partner violence in the radiology department}

The World Health Organization defines IPV as "any behavior within an intimate relationship that causes physical, psychological or sexual harm to those in the relationship." These abusive acts range from physical and sexual violence to emotional abuse and controlling behavior, and can have a serious physical and emotional impact on the health of the victim, as outlined in a recent review [8]. While IPV may affect any person regardless of gender, religion, ethnicity, or socioeconomic status, several risk factors for IPV have been identified, including being female, aged under 65 years, or a single mother, and having a low income or a low educational status [9-12]. The risk of becoming a victim of IPV is further associated with mental health issues (e.g., adjustment disorders, intentional selfharm, and anxiety disorders), alcohol and substance abuse, and the immigration status of the victim [11, 13, 14]. The association of IPV with mental disorders and alcohol/ substance abuse is generally thought to be bidirectional, and can lead to or exacerbate serious mental health conditions (depression, post-traumatic stress disorder, or suicidal tendencies) [11]. Unemployment, problem drinking, exposure to violence as a child, patriarchal cultural norms, and impulsivity were identified as significant risk factors for males to engage in IPV against women [12, 13, 15]. The lifetime prevalence of IPV-related physical violence and/or unwanted sexual relations is $\sim 23 \%$ among women in the general population and $37-50 \%$ among women in primary care or EDs $[9,11]$. In a recent study in the USA, 57 per 100,000 ED visits by women were designated with a code relating to IPV [11]. However, other studies have reported that $1-7 \%$ of female patients present with acute IPV-related physical injuries in the ED, highlighting the prevalent underreporting of IPV [16].

A large retrospective study, in which ED visits were screened for IPV, revealed that over $80 \%$ of IPV victims were women, the victims had a median age of $\sim 30$ years, and $\sim 50 \%$ of the injuries occurred at home [17]. This study also showed that the most common IPV-related injuries were contusions/abrasions (43\%), lacerations (17\%), strains/sprains $(16 \%)$, internal organ injuries $(14 \%)$, and fractures (10\%) [17]. Most IPV fractures affect the face (nasal bones, orbits, maxillofacial bones, and skull), followed by the fingers and upper trunk (Fig. 1) [17-21].

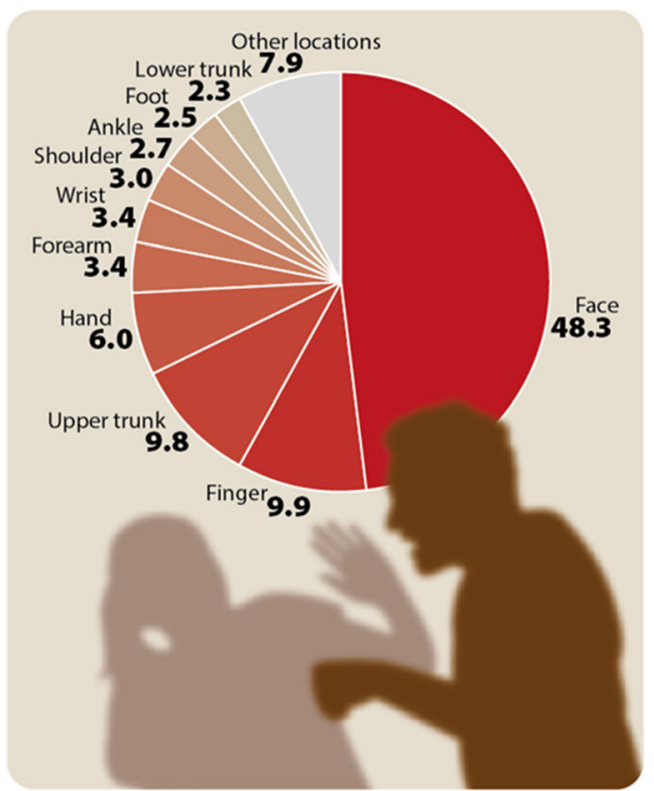

Fig. 1 The most common anatomic locations of fractures in IPV patients. Data from reference [17] 
Of note, the fractures most indicative of IPV are found in the upper and lower extremities, upper trunk, and head/neck [17]. Ulnar fractures, for instance, may point to a self-defense injury [22]. The IPV victim may selfreport that this fracture is related to a fall even though a radial fracture is the more likely outcome of such an accident [22]. In a recent study on the radiological profile of IPV victims, the most indicative radiological findings included soft tissue abnormalities (swelling, laceration, hematoma, or infection), musculoskeletal injuries (acute fractures, chronic or subacute fractures, or ligamentous injuries), and obstetric-gynecologic complications [18]. Furthermore, patient records may also provide critical information: the frequency of ED visits and imaging studies is higher for IPV victims than control populations, and the records may often contain terms related to contusions, abrasions, or bruising [18, 22-24]. As many IPV-related injuries may be difficult to discriminate from routine trauma, they can easily be overlooked or misinterpreted in a busy emergency or radiology department. Therefore, a meticulous anamnesis is essential to assess the possibility of IPV. In Fig. 2, we provide three exemplary IPV case reports.

In the future, artificial intelligence may help radiologists to identify IPV victims [22]. As the injuries may vary among different societal strata, demographic backgrounds, and cultures, it is important to include training sets of broad relevance with suitable control groups. In-depth studies will be needed to evaluate these approaches. As many IPV-related injuries appear similar to common accidents (e.g., those seen among elderly patients and alcoholics), these algorithms may need to include risk-related patient characteristics (age, medication, comorbidities, alcohol, and substance abuse). Importantly, communication between the radiologist and other caregivers with the suspected IPV victims is essential to assess the reasons for the trauma and to favorably impact patient outcomes.

\section{Procedure for radiologists when intimate partner violence is suspected}

Talking to a suspected IPV victim can be challenging and unsatisfying. We propose three principles for how to proceed in a case where there is a strong suspicion or a confirmed case (Fig. 3) $[8,22]$.

(1) It is important that the radiologist first communicates directly with the referring physician or care team when the mechanism of trauma is questionable or unclear to
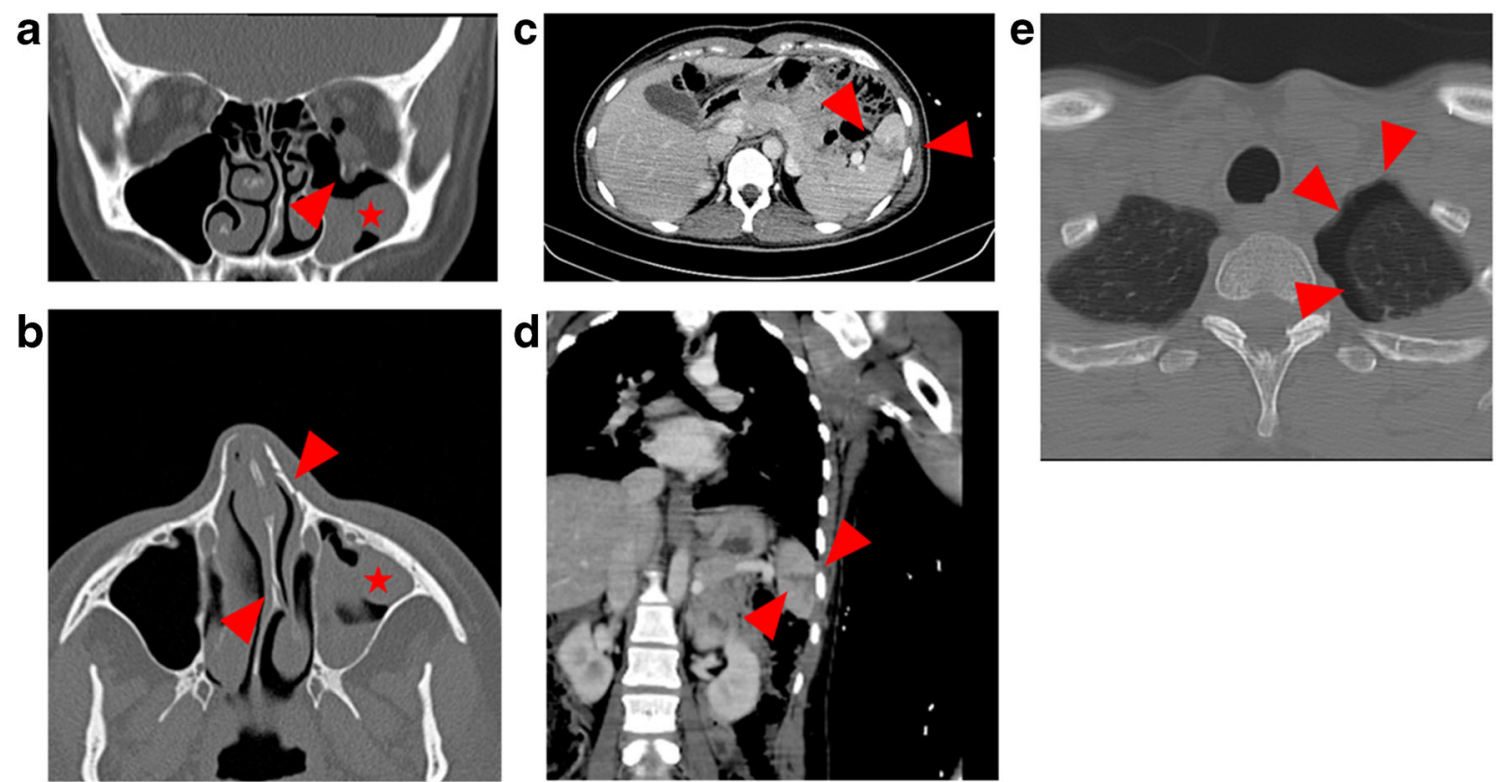

Fig. 2 Case 1: A 42-year-old female presenting with diplopia after she reported having been punched by her partner in the face. Head computed tomography (CT) revealed a left orbital floor fracture (arrowhead, a), blood-filled left maxillary sinus (asterisk, a, b), and nasal bone fractures as well as a nasal septum fracture (arrowhead, b) with deviation of the nasal septum to the left (arrowhead, b). The patient initially reported that she had run into a cupboard. Careful questioning indicated that she was attacked by her life partner. A social worker and, later, the police were informed. Case 2: After reporting a blunt trauma to the abdomen by her partner, abdominal CT of a 41-year-old female revealed splenic injury with parenchymal laceration (arrowheads) without intra-abdominal bleeding (c, d). The injury was treated conservatively and regularly checked by ultrasound. The bleeding stopped spontaneously. Police were informed. Case 3: A 49-year-old female patient was suspected to have been pushed by her partner, resulting in a fall against a bathtub. Caudal slices of a cervical spine CT showed incidental traumatic pneumothorax on the left side (arrowheads) as a result of rib fractures (not illustrated), but no pathology of the cervical spine (e). The police were informed. IPV was self-reported after questioning by medical professionals in these cases; however, we have no information on the outcomes of the police investigations on the alleged attacks 


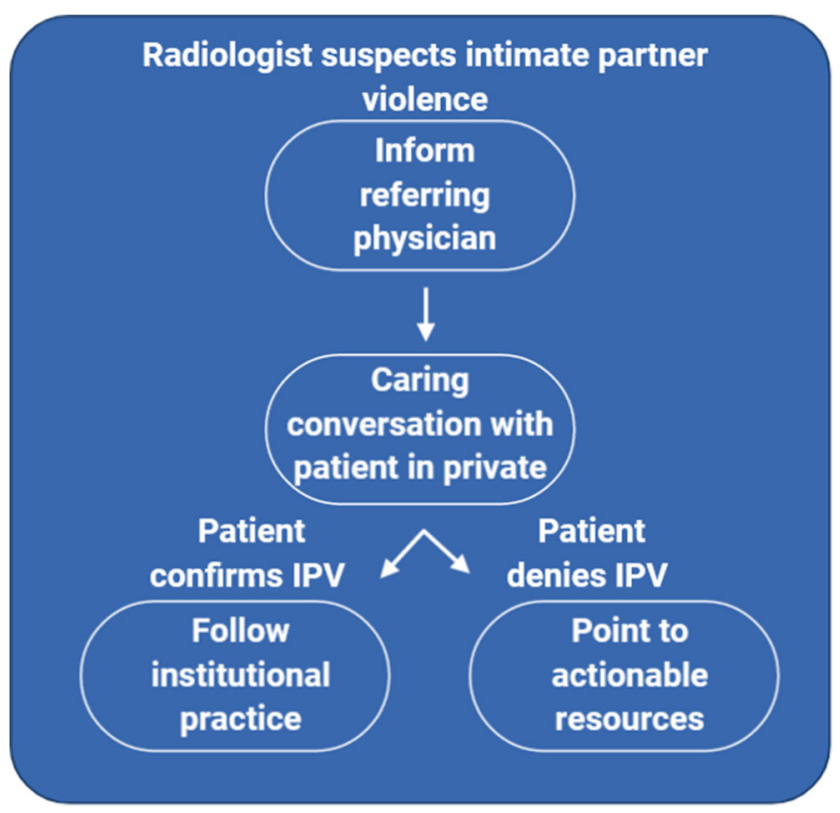

Fig. 3 Proposed procedure for radiologists in case of suspected IPV

the radiologist. There may already be further information available on the patient's background.

(2) When approaching the patient, it is crucial to create a safe and private space for the conversation. Even if the abuser is not present, the relationship of the accompanying person(s) to the abuser may impede the patient from disclosing any information.

(3) Patients must be informed that every health care provider is obliged to maintain confidentiality and the injured person should be assured that no information will be divulged beyond the medical team or automatically recorded in their medical files against the patient's wishes. However, as laws and policies regarding the obligation to report IPV differ between states and countries, health care providers should familiarize themselves with the standard practices of their institution on reporting of IPV. If a patient chooses to not disclose IPV, radiologists should respect their decision, but, as in cases with confirmed IPV, they should highlight actionable resources such as emergency services, hospital support programs, and IPV support organizations [25]. If it is not possible to hand the patient explicit brochures due to accompanying persons, telephone numbers, or QR codes with no obvious IPV-related information can be provided. Additionally, several apps are available for domestic violence victims, which offer a range of services such as IPV-evaluating questionnaires, support in setting up a safety plan, resourceful information, and live chat functions $[26,27]$. Certain apps are disguised as non-IPVrelated information (e.g., news or cooking sites) [26, 27]. If a patient discloses IPV and needs immediate assistance, radiologists should consult the social work team and treating physician, as per the standard practices of their institutions.

While radiologists and other physicians are understandably concerned about the health of the IPV victim, they should refrain from telling the victim what to do, as such behavior replicates the power and control patterns that IPV victims are exposed to in their abusive relationships. As physicians, we respect the patient's right to accept or refuse their doctor's advice. Just as a patient may refuse to undergo a proposed treatment, we must accept that an IPV victim may choose to walk out of the hospital with their abuser. It often takes several attempts for an IPV victim to recognize and disclose their circumstances and make a safety plan with their health care provider. Additionally, we should accept that we do not understand the entirety of the victim's situation, and the consequences that might arise from taking far-reaching decisions in that moment. However, if the physician suspects a direct threat to the life of the patient, informing the police or other authorities in accordance with local laws and regulations may be warranted.

\section{Radiologists: the new experts in domestic violence?}

Domestic violence remains taboo in many societies, and IPV victims are often stigmatized. These societal tendencies may also manifest in hospitals. Openly addressing IPV in the education of medical professionals will raise awareness of IPV as a serious health issue. IPV shares similarities with battered child syndrome, another societal taboo that is now more openly discussed in society and in hospitals, thanks to awareness campaigns. Radiologists have been making outstanding contributions to the health of physically abused children [28], and we believe that they can have a similarly positive influence on the lives of IPV victims. We acknowledge the "invisible wall" between diagnostic radiologists and patients, but we believe that radiologists will become more patient-centered in the future and contribute to patient care by directly engaging with their patients, particularly as artificial intelligence-based systems become more established [29-42]. As radiologists move beyond the reading room and join the multidisciplinary team providing direct care to the patient, they may choose to volunteer to handle IPV cases, especially if they are trained to handle the complexities of these conversations. Indeed, radiologists and radiographers are uniquely positioned to engage with IPV victims due to the physical separation between the victim and any accompanying persons in the radiology department. The diversity of radiological teams in terms of gender, ethnicity, and age may also be helpful in strengthening the rapport with the IPV victim [32-34]. Radiologists may further contribute to the field by studying injury patterns associated 
with IPV, especially in under-investigated subpopulations (e.g., the LGBT community, elderly, disabled individuals, pregnant women, and migrants) or different cultures.

\section{Conclusions}

In the light of the unprecedented social and economic crisis brought about by the COVID-19 pandemic, we urge radiologists and allied health professionals in the radiology department to be aware of IPV and carefully consider the provided history, even for common traumatic injuries. In the future, we expect radiologists to evolve further into patient-centered physicians who understand the complexities of patient care and proactively engage with their patients in caring conversations to identify and support IPV victims. In these times of unprecedented socioeconomic decline, social isolation, and the breakdown of support systems, we radiologists are at the frontline: we are witnessing the surge in IPV first-hand. In our opinion, it is essential that we turn from witnesses to helpers by letting IPV victims know that we care and that their lives matter.

Acknowledgments We thank women's shelters, helplines, hospitals, and the science community for their contributions to identifying and supporting IPV victims. Figure 3 was created using biorender. Institutional review board approval and informed consent was waived due to the retrospective character of this anonymized case series presented from the radiological image archive system of the University Medical Centre Mannheim, Germany.

Funding Bharti Khurana acknowledges funding provided by Gillian Reny Stepping Strong Innovator Award and BCRISP, Brigham Health. The other authors state that they have not received any funding related to this article.

\section{Compliance with ethical standards}

Guarantor The scientific guarantor of this publication is Andreas Gutzeit.

Conflict of interest The authors of this manuscript declare no relationships with any companies whose products or services may be related to the subject matter of the article.

Statistics and biometry No complex statistical methods were necessary for this paper.

Informed consent Written informed consent was not required for this study because of the anonymization of the case studies.

Ethical approval Institutional Review Board approval was waived.

\footnotetext{
Methodology

- Retrospective

- Case reports
}

\section{References}

1. Pfefferbaum B, North CS (2020) Mental health and the Covid-19 pandemic. N Engl J Med 383:510-512. https://doi.org/10.1056/ NEJMp2008017

2. Holmes EA, O'Connor RC, Perry VH et al (2020) Multidisciplinary research priorities for the COVID-19 pandemic: a call for action for mental health science. Lancet Psychiatry 7: $547-560$

3. Gutzeit A, Li Q, Matoori S, Li B, Wang L (2020) What can European radiologists learn from the outbreak of COVID-19 in China? A discussion with a radiologist from Wuhan. Eur Radiol 30 (7):3609-3611

4. Leslie E, Wilson R (2020) Sheltering in place and domestic violence: evidence from calls for service during COVID-19. J Public Econ 189:104241. https://doi.org/10.2139/ssrn.3600646

5. Mazza M, Marano G, Lai C, Janiri L, Sani G (2020) Danger in danger: Interpersonal violence during COVID-19 quarantine. Psychiatry Res 289:113046. https://doi.org/10.1016/j.psychres. 2020.113046

6. Bradbury-Jones C, Isham L (2020) The pandemic paradox: The consequences of COVID-19 on domestic violence. J Clin Nurs 29:2047-2049. https://doi.org/10.1111/jocn.15296

7. Usher K, Bhullar N, Durkin J, Gyamfi N, Jackson D (2020) Family violence and COVID-19: increased vulnerability and reduced options for support. Int J Ment Health Nurs 29:549-552. https://doi. org/10.1111/inm.12735

8. Miller E, McCaw B (2019) Intimate partner violence. N Engl J Med 380:850-857. https://doi.org/10.1056/NEJMra1807166

9. Breiding MJ, Black MC, Ryan GW (2008) Prevalence and risk factors of intimate partner violence in eighteen U.S. states/territories, 2005. Am J Prev Med 34:112-118. https://doi.org/10.1016/ j.amepre.2007.10.001

10. Thompson RS, Bonomi AE, Anderson M et al (2006) Intimate partner violence. Prevalence, types, and chronicity in adult women. Am J Prev Med 30:447-457. https://doi.org/10.1016/j.amepre. 2006.01.016

11. Beydoun HA, Williams M, Beydoun MA, Eid SM, Zonderman AB (2017) Relationship of physical intimate partner violence with mental health diagnoses in the nationwide emergency department sample. J Womens Health (Larchmt) 26:141-151. https://doi.org/10. 1089/jwh.2016.5840

12. Cunradi CB, Todd M, Duke M, Ames G (2009) Problem drinking, unemployment, and intimate partner violence among a sample of construction industry workers and their partners. J Fam Violence 24:63-74. https://doi.org/10.1007/s10896-008-9209-0

13. Chan KL (2012) Intimate partner violence in Hong Kong. In: Preventing family violence: a multidisciplinary approach. pp 19-57

14. Alisic E, Groot A, Snetselaar H, Stroeken T, van de Putte E (2017) Children bereaved by fatal intimate partner violence: a populationbased study into demographics, family characteristics and homicide exposure. PLoS One 12:e0183466. https://doi.org/10.1371/journal. pone. 0183466

15. Ozaki R, Otis MD (2017) Gender equality, patriarchal cultural norms, and perpetration of intimate partner violence: comparison of male university students in Asian and European cultural contexts. Violence Against Women 23:1076-1099. https://doi.org/10. 1177/1077801216654575

16. Anglin D, Sachs C (2003) Preventive care in the emergency department: screening for domestic violence in the emergency department. Acad Emerg Med 10:1118-1127. https://doi.org/10.1111/j. 1553-2712.2003.tb00585.x

17. Loder RT, Momper L (2020) Demographics and fracture patterns of patients presenting to US emergency departments for intimate 
partner violence. J Am Acad Orthop Surg Glob Res Rev 4: e2000009. https://doi.org/10.5435/jaaosglobal-d-20-00009

18. George E, Phillips CH, Shah N et al (2019) Radiologic findings in intimate partner violence. Radiology 291:62-69. https://doi.org/10. 1148/radiol.2019180801

19. Wu V, Huff H, Bhandari M (2010) Pattern of physical injury associated with intimate partner violence in women presenting to the emergency department: a systematic review and meta-analysis. Trauma Violence Abuse 11:71-82. https://doi.org/10.1177/ 1524838010367503

20. Hashemi HM, Beshkar M (2011) The prevalence of maxillofacial fractures due to domestic violence - a retrospective study in a hospital in Tehran, Iran. Dent Traumatol 27:385-388. https://doi.org/ 10.1111/j.1600-9657.2011.01016.x

21. Le BT, Dierks EJ, Ueeck BA, Homer LD, Potter BF (2001) Maxillofacial injuries associated with domestic violence. $J$ Oral Maxillofac Surg 59:1277-1283. https://doi.org/10.1053/joms. 2001.27490

22. Khurana B, Seltzer SE, Kohane IS, Boland GW (2020) Making the "invisible" visible: transforming the detection of intimate partner violence. BMJ Qual Saf 29:241-244

23. Kothari CL, Rhodes KV (2006) Missed opportunities: emergency department visits by police-identified victims of intimate partner violence. Ann Emerg Med 47:190-199. https://doi.org/10.1016/j. annemergmed.2005.10.016

24. Reis BY, Kohane IS, Mandl KD (2009) Longitudinal histories as predictors of future diagnoses of domestic abuse: modelling study. BMJ 339:849. https://doi.org/10.1136/bmj.b3677

25. Houry D, Kaslow NJ, Kemball RS et al (2008) Does screening in the emergency department hurt or help victims of intimate partner violence? Ann Emerg Med 51:433-442.e7. https://doi.org/10.1016/ j.annemergmed.2007.11.019

26. Brignone L, Edleson JL (2019) The dating and domestic violence app rubric: synthesizing clinical best practices and digital health app standards for relationship violence prevention smartphone apps. Int J Human Comput Interact 35:1859-1869. https://doi.org/10.1080/ 10447318.2019.1574100

27. Sinha S, Shrivastava A, Paradis C (2020) A survey of the mobile phone-based interventions for violence prevention among women. Adv Soc Work 19:493-517. https://doi.org/10.18060/22526

28. Nimkin K, Kleinman PK (1997) Imaging of child abuse. Pediatr Clin North Am 44:615-635. https://doi.org/10.1016/S00313955(05)70496-X

29. Recht M, Bryan RN (2017) Artificial intelligence: threat or boon to radiologists? J Am Coll Radiol 14:1476-1480. https://doi.org/10. 1016/j.jacr.2017.07.007

30. Gutzeit A, Fischmann A, Forstner R et al (2020) "I was seen by a radiologist, but unfortunately I can't remember the name and I still have questions. What should I do?" Radiologists should give thoughts to improve service professionalism and patient esteem. Cancer Imaging 20:1-8. https://doi.org/10.1186/s40644-020$0292-7$
31. Gutzeit A, Heiland R, Sudarski S et al (2019) Direct communication between radiologists and patients following imaging examinations. Should radiologists rethink their patient care? Eur Radiol 29: 224-231. https://doi.org/10.1007/s00330-018-5503-2

32. Arendt F, Karadas N (2019) Ethnic concordance in patientphysician communication: experimental evidence from Germany. J Health Commun 24:1-8. https://doi.org/10.1080/10810730.2018. 1549624

33. DiMatteo MR, Murray CB, Williams SL (2009) Gender disparities in physician-patient communication among African American patients in primary care. J Black Psychol 35:204-227. https://doi.org/ 10.1177/0095798409333599

34. Thornton RLJ, Powe NR, Roter D, Cooper LA (2011) Patientphysician social concordance, medical visit communication and patients' perceptions of health care quality. Patient Educ Couns 85:e201-e208. https://doi.org/10.1016/j.pec.2011.07.015

35. Matoori S, Froehlich JM, Breitenstein S et al (2019) Serum albumin, total bilirubin, and patient age are independent confounders of hepatobiliary-phase gadoxetate parenchymal liver enhancement. Eur Radiol 29 (11):5813-5822

36. Matoori S, Roveri M, Tiefenboeck P et. al (2019) An MRIguided HIFU-triggered wax-coated capsule for supertargeted drug release: a proof-of-concept study. Eur Radiol Exp 3 (1)

37. Matoori S, Froehlich JM, Breitenstein S et al (2016) Age dependence of spleen- and muscle-corrected hepatic signal enhancement on hepatobiliary phase gadoxetate MRI. Eur Radiol 26 (6):18891894

38. Gutzeit A, Matoori S, Froehlich JM et al (2016) Reduction in respiratory motion artefacts on gadoxetate-enhanced MRI after training technicians to apply a simple and more patient-adapted breathing command. Eur Radiol 26 (8):2714-2722

39. Sartoretti T, Sartoretti E, Bucher C et al (2017) Bacterial contamination of ultrasound probes in different radiological institutions before and after specific hygiene training: do we have a general hygienical problem?. Eur Radiol 27 (10):4181-4187

40. Gutzeit A, Matoori S, Froehlich J, Koh D (2016) Reduction in Respiratory Motion Artifacts on Gadoxetate Acid-enhanced MR Images after Training Technicians. Radiology 279 (3):981-982

41. Sudarski S, Haubenreisser H, Henzler T (2019) Incidence of transient interruption of contrast (TIC) - A retrospective single-centre analysis in CT pulmonary angiography exams acquired during inspiratory breath-hold with the breathing command: "Please inspire gently!". PLoS One 14(1):e0210473

42. Matoori S, Thian Y, Koh DM et al (2017) Contrast-Enhanced CT Density Predicts Response to Sunitinib Therapy in Metastatic Renal Cell Carcinoma Patients. Transl Oncol 10 (4):679-685

Publisher's note Springer Nature remains neutral with regard to jurisdictional claims in published maps and institutional affiliations. 